\title{
An Eco-Cruise Control for Electric Vehicles Moving on Slope Road with Constant Speed
}

\author{
Ying Zhang $\mathbb{D}^{1},{ }^{1}$ Yingjie Zhang $\mathbb{D},^{2}$ Zhaohua Liu $\mathbb{D}^{\circ},{ }^{3}$ Jinchao Chen $\mathbb{D}^{1},{ }^{1}$ Tao You $\mathbb{D}^{1},{ }^{1}$ \\ and Chenglie Du ${ }^{1}$ \\ ${ }^{1}$ School of Computer Science, Northwestern Polytechnical University, Xi'an 710129, China \\ ${ }^{2}$ College of Computer Science and Electronic Engineering, Hunan University, Changsha 410082, China \\ ${ }^{3}$ School of Information and Electrical Engineering, Hunan University of Science and Technology, Xiangtan 411201, China
}

Correspondence should be addressed to Ying Zhang; ying_zhang@nwpu.edu.cn

Received 10 July 2021; Revised 8 September 2021; Accepted 29 September 2021; Published 18 October 2021

Academic Editor: Peng Hang

Copyright (c) 2021 Ying Zhang et al. This is an open access article distributed under the Creative Commons Attribution License, which permits unrestricted use, distribution, and reproduction in any medium, provided the original work is properly cited.

The unreasonable actuation of electric vehicle's motor drive system usually results in a lot of unwanted energy consumption on a slope road. This paper proposes an eco-cruise control (ECC) scheme based on the driving condition estimation to decrease electric vehicle's energy consumption in the constant-speed cruise control mode. The eco-cruise control scheme is realized by reducing the unreasonable actuation of the motor drive system. The vehicle's total mass and pitch angle are estimated in real time by using an improved base-vector-based cross iteration estimator (BVCIE). Based on the estimated results, the required torque is predicted. Combining the speed deviation between the desired speed and the real speed, and the torque deviation between the required torque and the real torque, a three-power nonlinear controller of the ECC scheme is designed. The ECC scheme is validated on a slope road with different cruise speeds on a cosimulation platform, and the results indicate that the proposed strategy enjoys a better speed maintenance ability and energy efficiency compared with the benchmarked cruise control.

\section{Introduction}

In the last decades, the lane changing/lane keeping system $[1,2]$, antilock braking system [3], cruise control system (CCS) [4], advanced vehicle motion control systems [5-7], etc. have been widely implemented on the intelligent vehicles. Cruise control system is one of the first intelligent systems implemented on a vehicle, and it has been vastly applied to different types of ground vehicles. In the constantspeed cruise control mode, the driver can be disengaged from the control tasks of accelerator pedal and brake pedal, which helps to decrease driver's driving load so as to reduce traffic accidents. However, in the constant-speed cruise control mode, when a vehicle is moving on a complex road, such as on a slope road, the driving torque provided by the motor does not often meet the required torque, which is the unreasonable actuation phenomenon. The unreasonable actuation of the motor drive system usually causes the vehicle to consume a lot of unwanted energy. The unwanted energy consumption will undeniably decrease the vehicle's driving range, and this is one of the main factors hindering the spread and popularization of electric vehicles [8]. Therefore, improving energy efficiency is an urgent issue in the field of electric vehicles.

The past research on CCS mainly involves collision avoidance, constant-speed control, improvement of cruise system's fault tolerance, and other intelligent functions.

To address the collision problem, an intelligent cruise system is designed to force the vehicle acceleration to converge to the desired acceleration by using the on-board radar information [9]. In addition, [10, 11] propose advanced algorithms to address the problems of control strategy, scheduling, and real-time constraints simultaneously. Literature [12] initiates the longitudinal stopand-go cruise control system of heavy-duty trucks, and the test results show that the method not only meets the desired dynamic response, but also enjoys good robustness. In the parameters optimization aspect, $[13,14]$ 
propose different methods to select PID controller's parameters to improve the controller's performance with constant-speed control. The simulation results of the methods in $[13,14]$ show a good performance in vehicle's stability. To maintain the constant time headway with respect to the front vehicle, a neuro-fuzzy controller is proposed for intelligent cruise control of semiautonomous vehicles, and this method demonstrates better performance compared with the conventional PID controller [15]. In order to improve the fault tolerance of CCS, a prototype tool is designed in [16], and a fault recovery technique is proposed in [17] to improve the fault tolerance and robustness of CCS; these methods demonstrate a good performance in guaranteeing the normal work of CCS. In addition, to enable the driver to fully disengage himself/herself from the driving task, the new research on CCS is advancing in more intelligent directions, such as adaptive cruise control $[18,19]$ and cooperative adaptive cruise control [20-25].

Obviously, the aforementioned intelligent technologies and methods contribute to the development of the autonomous vehicles. However, most of the research has not considered the energy efficiency problem. In the last decades, energy consumption in the transportation field has sharply increased and has become one of the most serious challenges. In reality, the driving conditions greatly affect the vehicle's energy consumption [26].

Usually, the vehicle moving under traffic signs condition with cruise mode will increase energy consumption level due to the unreasonable start, stop, acceleration, and deceleration. To reduce the idle time at a red light and decrease energy consumption, a predictive cruise control [27] that uses the information of upcoming traffic lights is proposed to judge a predictive manner. By this means, it contributes to the improvement of the energy efficiency of the vehicle's powertrain. According to the traffic information, the energyefficient cruise control systems [28, 29] are designed to optimize the acceleration profile so as to minimize energy consumption. Similarly, [30] designs an adaptive cruise control system based on the upcoming traffic signal information to reduce the idle time at stop lights and fuel consumption. To decrease the energy consumption due to the queue effects at a signalized intersection, [31] proposes an eco-cooperative adaptive cruise control to improve the vehicle's fuel efficiency, and the simulation result demonstrates that the overall fuel consumption could be saved up to $40 \%$.

The latest research indicates that driving conditions such as the road slope, the vehicle's pitch angle, and the vehicle's mass are seriously affecting the energy consumption and proposes many excellent strategies to improve the energy efficiency [32-36]. However, these methods obtain the road profile from digital maps or from global positioning system (GPS) [32-34], and this will have poor performance in case of shelter conditions. In addition, most of the research mainly focuses on the fuel vehicles [34-36]. Compared with the fuel vehicles, the electric vehicles call for more urgent need to address the energy efficiency problem because of the limits of the driving range.
Therefore, in this paper, we specifically concentrate on the improvement of the electric vehicle's energy efficiency in constant-speed cruise control mode on slope road. Different from the prevailing methods, in this paper, a novel ECC scheme is proposed without using the digital map's information or the GPS information. To guarantee that the electric vehicle has good speed maintenance ability and energy efficiency performance in constant-speed cruise control mode, the ECC scheme is realized by considering the deviation in vehicle speed and the deviation in the required torque. With the ECC scheme, the driving torque and the braking torque are optimized so as to reduce the unreasonable actuation of the motor drive system. In summary, the major innovations and significance of this paper are as follows:

(1) There is methodological innovation in decreasing electric vehicle's energy consumption in the constant-speed cruise control mode on slope road. In this paper, an ECC strategy is proposed to improve the speed maintenance ability and energy efficiency performance. With the ECC strategy, the average driving efficiency and the average regenerative braking efficiency of the motor drive system can be improved.

(2) An improved BVCIE is proposed to estimate the vehicle's driving load factors (total mass and pitch angle). In the framework of the improved BVCIE, a new sliding-model-based strategy is designed to improve the robustness of the estimator. With the sliding-model-based strategy, the phenomenon of signal distortion and data loss can be compensated for.

(3) A new three-power nonlinear controller is designed based on the estimated driving load factors to avoid the unreasonable actuation of the motor's torque caused by the slope road condition. The controller enables the motor drive system to provide reasonable driving torque and braking torque according to the deviations in the vehicle speed and the desired torque. Therefore, the unwanted actuation can be avoided.

The rest of this paper is organized as follows. In Section 2, the vehicle dynamics is analyzed, and the required torque is presented. In Section 3, the vehicle's total mass and the pitch angle are estimated by an improved BVCIE. In Section 4, a novel ECC scheme is proposed. In the ECC scheme, a new controller is designed, and its stability is proved. In Section 5, the simulation platform is introduced, and the estimation results of vehicle's mass and pinch angle are presented. In Section 6, the cruise control performances by the proposed ECC scheme and by the benchmarked cruise control are analyzed in detail. In Section 7 , the energy consumption and the energy saving performance of the proposed ECC and the benchmarked cruise control are analyzed at different cruise speeds. Finally, we conclude this paper with its main novelties and contributions in Section 8. 


\section{Vehicle Dynamics}

When an electric vehicle is moving on a slope road in the constant-speed cruise control mode, the required torque in the longitudinal motion is a real-time variable due to the variation of the driving conditions, such as the vehicle's pitch angle and the longitudinal speed. Therefore, to maintain the vehicle at the desired cruise speed, the CCS needs to control the driving torque and the braking torque according to the driving conditions. In vehicle's moving process, the factors affecting the vehicle's performance include the rolling resistance, the equivalent aerodynamic drag resistance, the slope resistance, and the acceleration resistance, as shown in Figure 1.

Assuming the vehicle's total mass is $m$ and the longitudinal acceleration is $\dot{v}$, the longitudinal dynamics model can be expressed as

$$
F=F_{\text {rol }}+F_{\text {aero }}+F_{\text {slope }}+m \dot{v},
$$

where $F$ represents a resultant force of the driving force and the braking force, $F_{\text {rol }}$ refers to the rolling resistance, $F_{\text {aero }}$ refers to the equivalent aerodynamic drag resistance, and $F_{\text {slope }}$ refers to the slope resistance. In (1), the rolling resistance depends on the road condition and the vehicle's total mass, which can be expressed as follows:

$$
F_{\text {rol }}=m g \mu \cos \theta,
$$

where $g$ is the acceleration due to gravity, $\mu$ refers to the rolling resistance coefficient, and $\theta$ refers to the vehicle's pitch angle.

The slope resistance in (1) is affected by the factors of the vehicle's total mass and the pitch angle, and this relationship can be expressed as follows:

$$
F_{\text {slope }}=m g \sin \theta .
$$

The equivalent aerodynamic drag resistance in (1) can be expressed as

$$
F_{\text {aero }}=\frac{1}{2} A C_{d} \rho v^{2},
$$

where $A$ is the frontal area of the vehicle, $C_{d}$ is the aerodynamic drag coefficient, $\rho$ is the density of air, and $v$ is the vehicle speed. Assuming the wheel's radius is $r$, the resultant torque $T$ in the longitudinal motion can be expressed as

$$
T=F r .
$$

In (5), the resultant torque is from the electric motor (EM). The relation between the resultant torque and the motor's torque can be depicted as

$$
T_{m}=\frac{T}{i_{g} \eta_{g}},
$$

where $i_{g}$ refers to the gear ratio, $\eta_{g}$ refers to the efficiency of the mechanical system, and $T_{m}$ is the motor's torque.

When a vehicle is moving in the constant-speed cruise control mode, the ideal condition is the vehicle moving at the desired cruise speed and the acceleration equal to zero. In

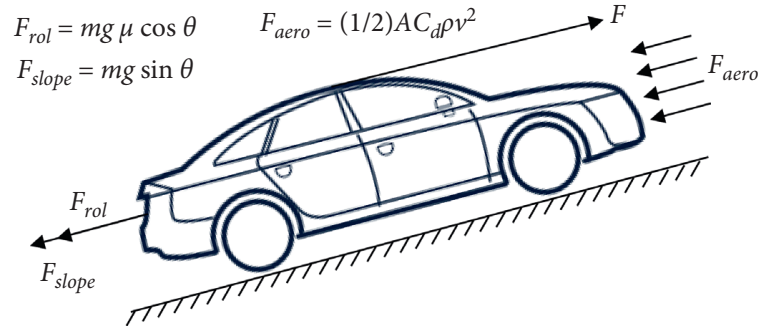

Figure 1: The diagram of vehicle longitudinal dynamics.

addition, in the real condition, the vehicle's total mass is affected by the passenger number and the payload state, and the pitch angle is determined by the real-time road condition. Therefore, the vehicle's total mass is an unknown parameter in the moving process, and the pitch angle is a variable in the moving process. Assuming the total mass can be estimated with $\widehat{m}$ in real time and the vehicle's pitch angle can be estimated with $\widehat{\theta}$ in real time, the required resultant force at the sampling instance $t_{k}$ can be expressed as

$$
\begin{aligned}
F_{r}\left(t_{k}\right) & =F_{\text {rol }}\left(t_{k}\right)+F_{\text {aero }}\left(t_{k}\right)+F_{\text {slope }}\left(t_{k}\right) \\
& =\widehat{m}\left(t_{k}\right) g \mu \cos \left(\hat{\theta}\left(t_{k}\right)\right)+\hat{m}\left(t_{k}\right) g \sin \left(\hat{\theta}\left(t_{k}\right)\right)+0.5 A C_{d} \rho v_{r}^{2} \\
& =\widehat{m}\left(t_{k}\right) g \sqrt{1+\mu^{2}} \sin \left(\widehat{\theta}\left(t_{k}\right)+\theta_{\mu}\right)+0.5 A C_{d} \rho v_{r}^{2} .
\end{aligned}
$$

In (7), $F_{r}$ represents the desired resultant force, $v_{r}$ represents the desired cruise speed in the constant-speed cruise control mode, and $\theta_{\mu}$ can be calculated by $\theta_{\mu}=\arctan \mu$.

According to (5) and (7), the required torque $T_{r}$ under the condition that the acceleration is equal to zero can be expressed as

$$
T_{r}\left(t_{k}\right)=F_{r}\left(t_{k}\right) r
$$

\section{Estimation of the Total Mass and Pitch Angle}

3.1. Estimator Design. The real-time vehicle speed in constant-speed cruise control mode is affected by the driving condition, and consequently when calculating the real-time required torque, it needs to obtain the real-time variables of the vehicle's total mass and the vehicle's pitch angle. To address this problem, in this paper, we adopt a BVCIE because it does not need to reconstruct a state space equation. More importantly, compared with Kalman filter (KF), recursive least square (RLS) method, etc., the BVCIE enjoys a higher estimation accuracy. In addition, compared with the neural network method, the BVCIE does not need to train the model through a large amount of data. To construct the BVCIE, we define $y=\dot{v}, \varphi_{1}=T / r-0.5 \rho A C \mathrm{~d} v^{2}$, $\varphi_{2}=-g\left(1+\mu^{2}\right)^{1 / 2}$; the vehicle longitudinal dynamics (1) can be rewritten with a linear formula:

$$
y=\phi x .
$$

In (9), $x$ is the vector which needs be estimated, $x=\left[x_{1}\right.$, $\left.x_{2}\right]^{T} \in \mathbb{R}$, which can be written as follows: 


$$
\left\{\begin{array}{l}
x_{1}=\frac{1}{m} \\
x_{2}=\sin \left(\theta+\theta_{\mu}\right) .
\end{array}\right.
$$

Assuming the vector of $x$ can be estimated with $\hat{x}(k)$ $k-1)$, where $k$ and $k-1$ are the indexes of the discrete sampling instants, we can easily obtain the system's estimated output according to (9), and it can be expressed as follows:

$$
\widehat{y}(k)=\phi(k) \hat{x}\left(\frac{k}{k-1}\right),
$$

where $\hat{x}(k / k-1)$ represents the estimated vector by a cross iteration strategy and $\hat{y}$ is the estimated output. Based on (9) and (11), we can design the estimator with the following formulation:

$$
\widehat{x}(k)=\widehat{x}\left(\frac{k}{k-1}\right)+\phi^{-1}(k)(y(k)-\widehat{y}(k)) .
$$

According to (9) and (10), the $\widehat{x}_{1}(k / k-1)$ can be formulated by a cross iteration strategy:

$$
\widehat{x}_{1}\left(\frac{k}{k-1}\right)=f_{1}(k)+g_{1}(k) \widehat{x}_{2}\left(\frac{k}{k-1}\right),
$$

where $f_{1}(k)=\dot{v}(k) /\left((T(k) / r)-\left((1 / 2) \rho A C_{d} v^{2}(k)\right)\right), g_{1}(k)$ $=\left(g \sqrt{1+\mu^{2}}\right) /\left((T(k) / r)-\left((1 / 2) \rho A C_{d} v^{2}(k)\right)\right)$.

In vehicle's moving process, the total mass of the vehicle can be deemed as a constant; thus, we can formulate the following equation:

$$
\widehat{x}_{1}\left(\frac{k}{k-1}\right)=\widehat{x}_{1}\left(\frac{k-1}{k-2}\right)=\widehat{x}_{1}(k-1) .
$$

Assuming $\hat{x}_{1}(k-1)$ can be known in advance, $\widehat{x}_{2}(k / k-1)$ can be formulated using a cross iteration strategy:

$$
\begin{aligned}
\widehat{x}_{2}\left(\frac{k}{k-1}\right) & =f_{2}(k)+g_{2}(k) \widehat{x}_{1}\left(\frac{k}{k-1}\right) \\
& =f_{2}(k)+g_{2}(k) \widehat{x}_{1}\left(\frac{k-1}{k-2}\right) \\
& =f_{2}(k)+g_{2}(k) \widehat{x}_{1}(k-1),
\end{aligned}
$$

where $f_{2}(k)=-\left(\dot{v}(k) /\left(g \sqrt{1+\mu^{2}}\right)\right), \quad g_{2}(k)=((T(k) / r)-$ $\left.\left((1 / 2) \rho A C_{d} v^{2}(k)\right)\right) /\left(g \sqrt{1+\mu^{2}}\right)$.

For the proof of its convergence, please refer to [37] for the details. Now, the vehicle's total mass and the vehicle's pitch angle can be estimated by the above estimator. In the above estimator, the inputs of the estimator are the resultant torque, the vehicle speed, and the acceleration. The outputs of the estimator are the estimated vehicle's total mass and the estimated vehicle's pitch angle, as shown in Figure 2.

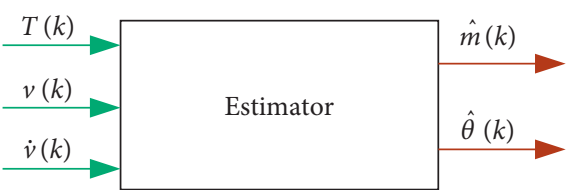

FIgURE 2: The estimator's diagram for estimating vehicle's total mass and pitch angle.

3.2. Sliding-Mode-Based Strategy. Actually, the input signals of the estimator are bounded by a physical constraint, and this constraint can be described as

$$
\left\{\begin{array}{l}
T \in\left[T_{\min }, T_{\max }\right], \\
v \in\left[v_{\min }, v_{\max }\right], \\
\dot{v} \in\left[a_{\min }, a_{\max }\right],
\end{array}\right.
$$

where min and max refer to the minimum value and maximum value, respectively. In the vehicle system, the disturbances due to the internal and external factors and the vehicle vibration could result in the input signals' distortion and even the measurement data's loss [38], shown as region 1 in Figures 3(a) and 3(b) and region 2 in Figure 3(c), respectively. These cases will seriously affect the estimation results and even result in the wind-up of the estimator. To fix this problem, a sliding-mode-based strategy for processing the input signals is proposed.

The sliding-mode-based strategy includes a look-backward method and a double-threshold method. When data get lost in an input signal, the look-backward method is put into action to fill in the lost data, as shown in the flow diagrams in Figures 3(e) and 3(g). In the look-backward method, a batch of the input data sequence can be defined as

$$
\ell=\left[\ell\left(t_{k-2}\right) \ell\left(t_{k-1}\right) \ell\left(t_{k}\right)\right] .
$$

When the input data get lost at the sampling instance $t_{k}$, the filling value with the look-backward method can be expressed as

$$
\begin{aligned}
\ell\left(t_{k}\right) & =\ell\left(t_{k-1}\right)+\Delta \ell\left(t_{k-1}\right) \\
& =\ell\left(t_{k-1}\right)+\left(\ell\left(t_{k-1}\right)-\ell\left(t_{k-2}\right)\right) \\
& =2 \ell\left(t_{k-1}\right)-\ell\left(t_{k-2}\right) .
\end{aligned}
$$

Furthermore, when the input signals are greater than its maximum thresholds or smaller than its minimum thresholds, the double-threshold method is applied to process the input signals, as in the flow diagrams shown in Figures 3(d) and 3(f). The double-threshold method can be expressed as follows:

$$
\ell= \begin{cases}\ell_{\max }, & \ell \geq \ell_{\max }, \\ \ell_{\min }, & \ell \leq \ell_{\min } .\end{cases}
$$

With the above signal processing strategy, the robustness of the estimator can be enhanced. 


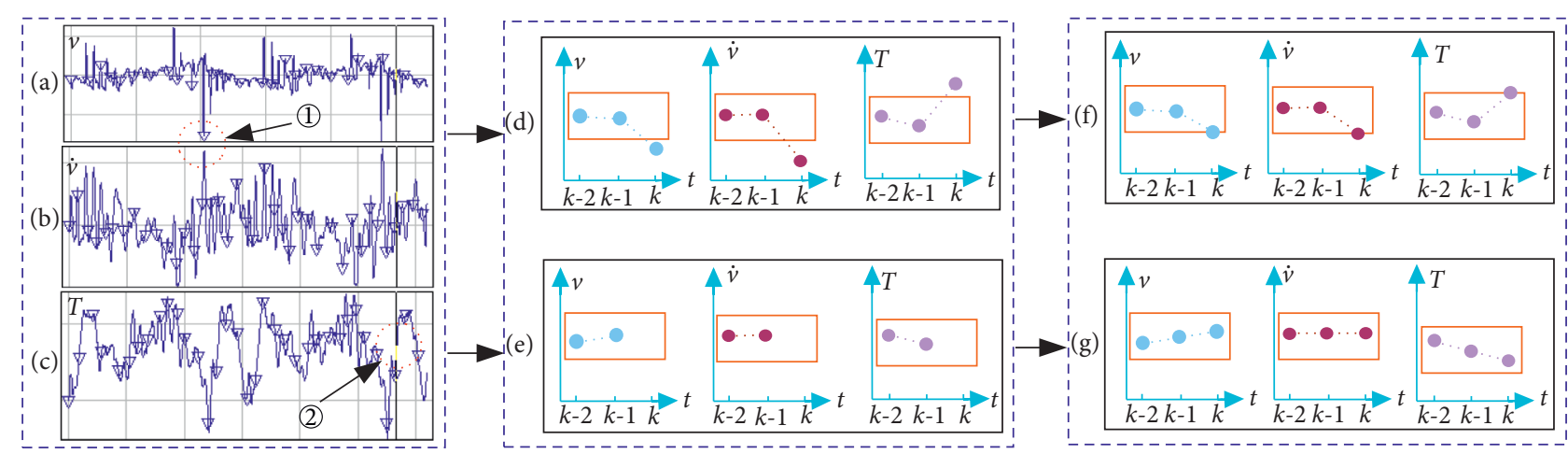

FIgURE 3: The flow diagram of the sliding-mode-based strategy.

\section{Eco-Cruise Control}

4.1. Control Scheme. The required torque in (8) in Section 2 represents the torque in the condition that the vehicle maintains the desired constant speed. However, when the vehicle is moving on a slope road, the vehicle speed usually shows fluctuations. In this condition, the CCS needs to regulate the driving torque and the braking torque so as to guarantee that the speed maintains the required value. On the sloping road, the driving load is a variable because the road slope is a variable. Thus, in the vehicle speed regulation process, unreasonable actuation conditions in the driving torque and braking torque may occur due to the slope road condition, which will result in a lot of unwanted energy consumption. For this problem, this section proposes an ECC scheme to reduce the unreasonable actuations of the driving torque and braking torque. In order to clearly describe the ECC scheme, the traditional cruise control scheme and the ECC scheme are shown in Figure 4.

Figure 4(a) shows the traditional cruise control scheme. This scheme only considers the deviation in the speed $(e)$ and does not consider the desired driving torque. For the cruise control scheme in Figure 4(a), a state-of-the-art method is model predictive control (MPC). For the MPC-based cruise control, please refer to $[23,28,39,40]$ for details. Figure 4(b) shows the ECC-based cruise control scheme. As shown in Figure 4(b), the controller has two inputs: one is the deviation in the speed $(e)$, and the other is the deviation in the required torque $(\Delta T)$. Hence, the ECC scheme can not only guarantee that the speed maintains the desired value, but also keep the driving torque and the braking torque away from the unreasonable actuation.

4.2. Controller Design and Stability Analysis. In order to design a controller for the ECC scheme, the control system of the electric vehicle in Section 2 can be depicted as

$$
\left\{\begin{array}{l}
\dot{\mathbf{x}}=\boldsymbol{\alpha} \mathbf{x}^{2}+\mathbf{u}-\mathbf{b} \\
\mathbf{y}=\mathbf{x}
\end{array}\right.
$$

where $\quad \mathbf{x}=v, \quad \mathbf{u}=T /(m \cdot r), \quad \alpha=-0.5 \rho A C_{d} / m$, $\mathbf{b}=g(\sin \theta+\mu \cos \theta)$. To depict the output deviation of the control system (20), the tracking error in the output $\left(\mathbf{e}_{o}\right)$ can be defined as

$$
\mathbf{e}_{o}=\mathbf{y}_{r}-\mathbf{y} .
$$

In addition, for the control system (20), the tracking error in the input $\left(\mathbf{e}_{i}\right)$ can be depicted as

$$
\mathbf{e}_{i}=\mathbf{u}_{r}-\mathbf{u} .
$$

In (22), ur $=T_{r} /(m \cdot r)$, and $T_{r}$ can be obtained by (8). According to (21) and (22), when considering the errors in the input and output, a three-power nonlinear controller can be designed as in the following formulation:

$$
\mathbf{u}=\mathbf{K}_{o} \mathbf{e}_{o}^{3}+\mathbf{K}_{i} \mathbf{e}_{i}
$$

where $\mathbf{K}_{o}$ refers to the gain of the output $\mathbf{y}$, and $\mathbf{K}_{i}$ refers to the gain of the required input $\mathbf{u}$.

Theorem 1. For the control system (20), if there exist $\boldsymbol{K}_{o} \in\left[0, \xi_{1}\right]$ and $K_{i} \in\left[0, \xi_{2}\right]$, and they satisfy the following condition:

$$
3 \mathbf{K}_{o} \mathbf{e}_{o}^{2} \dot{\mathbf{e}}_{o}+\mathbf{K}_{i} \dot{\mathbf{e}}_{i}-\dot{\mathbf{b}}=\ddot{\mathbf{y}}-2 \boldsymbol{\alpha} \dot{\mathbf{x}} \mathbf{y}_{r},
$$

then the output $\mathbf{y}$ will converge to $\mathbf{y}_{r}$ by the control strategy (23).

Proof. If the output $\mathbf{y}$ converges to $\mathbf{y}_{r}$, (21) should satisfy $\mathbf{e}_{o} \longrightarrow 0$ by the control strategy (23). Equation (20) can be differentiated as

$$
\begin{aligned}
& \ddot{\mathbf{x}}=2 \mathbf{\alpha x} \dot{\mathbf{x}}+\dot{\mathbf{u}}-\dot{\mathbf{b}} \\
& =2 \mathbf{\alpha x} \dot{\mathbf{x}}+3 \mathbf{K}_{o} \mathbf{e}_{o}^{2} \dot{\mathbf{e}}_{o}+\mathbf{K}_{i} \dot{\mathbf{e}}_{i}-\dot{\mathbf{b}} .
\end{aligned}
$$

Substituting (25) into (21) yields

$$
\mathbf{e}_{o}=\mathbf{y}_{r}-\frac{(\ddot{\mathbf{x}}+\dot{\mathbf{b}})}{2 \boldsymbol{\alpha} \dot{\mathbf{x}}}-\frac{\left(3 \mathbf{K}_{o} \mathbf{e}_{o}^{2} \dot{\mathbf{e}}+\mathbf{K}_{i} \dot{\mathbf{e}}_{i}\right)}{2 \boldsymbol{\alpha} \dot{\mathbf{x}}} .
$$

According to condition (24), $\ddot{\mathbf{y}}$ can be expressed as

$$
\ddot{\mathbf{y}}=3 \mathbf{K}_{o} \mathbf{e}_{o}^{2} \dot{\mathbf{e}}_{o}+\mathbf{K}_{i} \dot{\mathbf{e}}_{i}-\dot{\mathbf{b}}+2 \boldsymbol{\alpha} \dot{\mathbf{x}} \mathbf{y}_{r} .
$$

Following the output equation in (20), one can obtain

$$
\ddot{\mathbf{y}}=\ddot{\mathbf{x}} \text {. }
$$

Thus, (26) can be rewritten as

$$
\ddot{\mathbf{x}}=3 \mathbf{K}_{o} \mathbf{e}_{o}^{2} \dot{\mathbf{e}}+\mathbf{K}_{i} \dot{\mathbf{e}}_{i}-\dot{\mathbf{b}}+2 \boldsymbol{\alpha} \dot{\mathbf{x}} \mathbf{y}_{r} .
$$

Substituting (29) into (26) yields $\mathbf{e}_{o}=0$. 


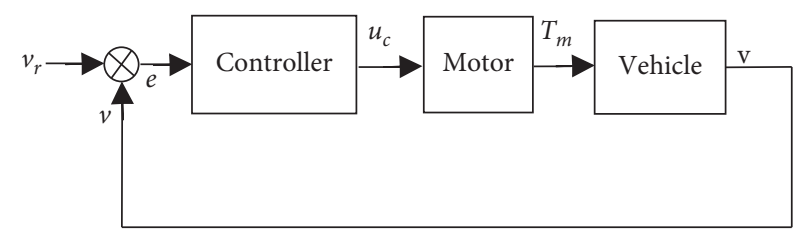

(a)

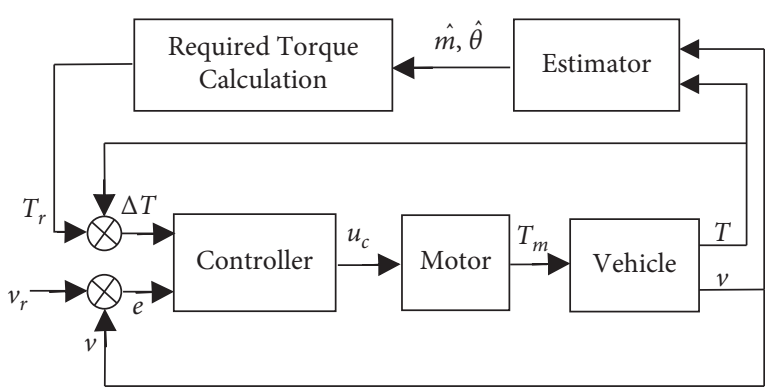

(b)

Figure 4: Cruise control scheme. (a) Traditional cruise control scheme. (b) Eco-cruise control scheme.

\section{Simulation Environment and Estimation Results}

This section first presents the test vehicle and the driving environment; then, the state estimation results and the control performance are presented with detailed analysis.

\subsection{The Test Vehicle and the Test Road. In this work, the ECC} is validated on a cosimulation platform with CarSim and Matlab/Simulink. The vehicle simulator combining CarSim with Matlab is a high-fidelity platform for validating the vehicle dynamics and control methods. The vehicle's model in CarSim is close to the real-world vehicle. More importantly, the validation through CarSim and Matlab can effectively avoid the influence from the traffic condition and driver's behavior. For the cosimulation platform in this paper, the version of the Matlab is R2007a, and the version of the CarSim is 2016.0. The parameters of the vehicle body and road information are set in CarSim, and the electric powertrain, the proposed ECC, and the benchmarked method are built in Matlab/Simulink. The cosimulation platform is validated on personal computer with Windows 10 64-bit operating system, $16 \mathrm{G}$ memory, i7-10510U CPU, $2.30 \mathrm{GHz}$.

The simulated vehicle is a front wheel drive electric vehicle, as shown in Figure 5(a). The motor drive system of this vehicle is shown in Figure 5(b). In driving mode, the battery provides power for the EM, and the EM drives the front wheels. The EM is a direct-current motor, and the gearbox has single variable speed ratio. In the driving mode, the battery provides energy to the electric powertrain, and in the braking mode, the regenerative braking energy is fed back to the battery. An asphalt road is selected as the test road, which is shown in Figure 5(c). The profile of the road is from the real world. The length of the test road is about 275 meters, the maximum height of the test road is about 4.173 meters, and the test road includes uphill parts and downhill parts. The profile of the test road is shown in Figure 5(d). The driving environment, the designed parameters, and the specification of the test vehicle are shown in Table 1.

In order to analyze the efficiency of the motor, the efficiency map of the motor should be built. The accurate motor's efficiency map is related to motor's torque and speed. However, as the efficiencies are similar within a relative large range of motor rotational speeds, a simplified efficiency map can be fitted by only considering the motor's torque, and this simplified efficiency map has been successfully used in [41]. For this reason, in this paper, we regard the motor efficiency as a function of torque. The driving efficiency and regenerative braking efficiency of the EM can be fitted by the least square method. Assume that the curve function of the driving efficiency and regenerative braking efficiency can be expressed as

$$
y=a_{4} x^{4}+a_{3} x^{3}+a_{2} x^{2}+a_{1} x^{1}+a_{0} .
$$

To identify the parameters $a_{0}, a_{1}, a_{2}, a_{3}, a_{4}$ based on the least square method, we need to measure several groups' data of the motor's torque and motor efficiency. Since the motor's torque, the motor's rotational speed, and the input/ output power of the battery in driving mode and braking mode can be easily collected, the motor efficiency (including the driving efficiency and the regenerative braking efficiency) can be calculated with the following equation: where $\eta_{o}$ refers to the driving efficiency, $\eta_{i}$ refers to the regenerative braking efficiency, $\omega$ is the motor's rotational speed, and $P_{i} / P_{o}$ refers to the input/output power of battery.

In order to derive the motor efficiency map, we calculated 11 groups of the driving efficiencies and 11 groups of the regenerative braking efficiencies based on (31). Thus, the curves of the driving efficiency and the regenerative braking efficiency can be fitted. For the fitted driving efficiency curve, $a_{0}=0.1311, a_{1}=0.0224, a_{2}=-2.3647 \times 10^{-4}, a_{3}=9.8593 \times$ $10^{-7}, a_{4}=-1.4736 \times 10^{-9}$. For the fitted braking efficiency curve, $\quad a_{0}=-8.697 \times 10^{-4}, \quad a_{1}=0.0125, a_{2}=-8.685 \times 10^{-5}$, $a_{3}=2.5372 \times 10^{-7}, a_{4}=-2.6187 \times 10^{-10}$. The calculated driving efficiency, the regenerative braking efficiency, and the fitted curves are shown in Figure 6. The discrete red points are the calculated driving efficiency, and the dashed green curve refers to the fitted driving efficiency. The discrete blue points refer to the calculated regenerative braking efficiency, and the solid magenta curve refers to the fitted braking regenerative efficiency. In the braking mode, part of the mechanical energy will be converted to the thermal energy and get lost. In addition, the efficiency of the power conversion circuit is unlikely to reach $100 \%$. Moreover, during the braking process, part of the regenerative electric energy will get lost. For these reasons, in Figure 6, the regenerative braking efficiency is lower than the driving efficiency under the same torque condition. 


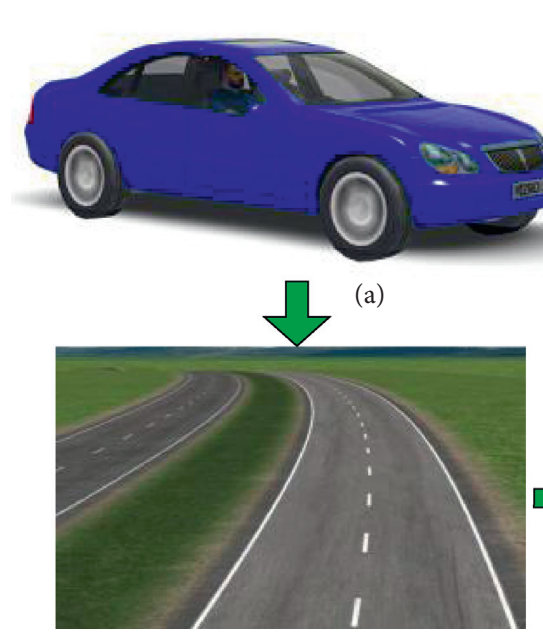

(c)
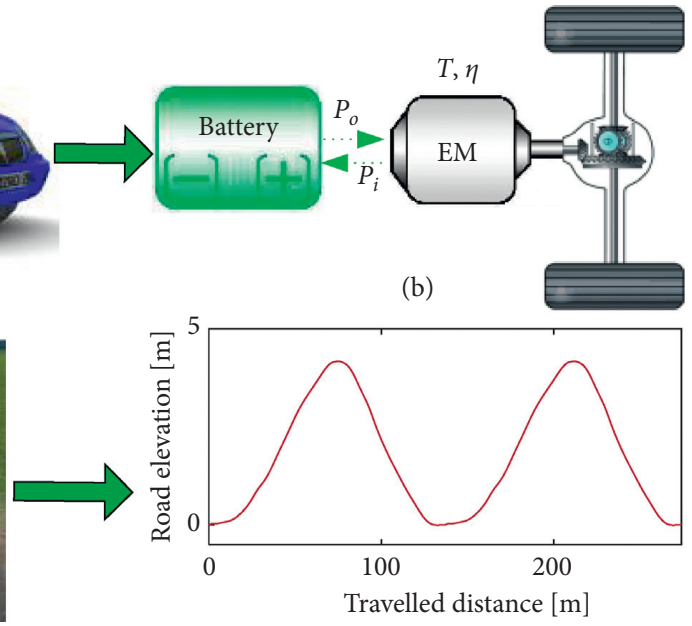

(d)

FIgURE 5: Diagram of the test vehicle and the test road.

TABle 1: Parameters and specification of the test vehicle.

\begin{tabular}{lccc}
\hline Name & Symbol & Values & Unit \\
\hline Vehicle mass & $m$ & 1450 & $\mathrm{~kg}$ \\
Air density & $\rho$ & 1.206 & $\mathrm{~N} \cdot \mathrm{s}^{2} / \mathrm{m}^{4}$ \\
Maximum road elevation & $h$ & 4.173 & $\mathrm{~m}$ \\
Vehicle front area & $A$ & 2.2 & $\mathrm{~m}$ \\
Wheel radius & $r$ & 0.353 & $\mathrm{~m}$ \\
Roll inertia & $I_{x x}$ & 536.6 & $\mathrm{mg} \cdot \mathrm{m}^{2}$ \\
Pitch inertia & $I_{y y}$ & 1536.7 & $\mathrm{~kg} \cdot \mathrm{m}^{2}$ \\
Yaw inertia & $I_{z z}$ & 1536.7 & $\mathrm{~kg} \cdot \mathrm{m}^{2}$ \\
Road distance & $l$ & 275 & $\mathrm{~m}$ \\
Battery voltage & $V_{\text {bat }}$ & 335 & $\mathrm{~V}$ \\
Battery capacity & $E_{\text {bat }}$ & 61 & $\mathrm{kwh}$ \\
EM's maximum torque & $T_{m-\max }$ & 320 & $\mathrm{Nm}$ \\
EM's maximum power & $P_{m-\text { max }}$ & 160 & 7.6 \\
Gear ratio & $i_{g}$ & 0.28 & - \\
Drag coefficient & $C_{d}$ & 0.012 & - \\
Rolling friction coefficient & $\mu$ & - \\
\hline
\end{tabular}

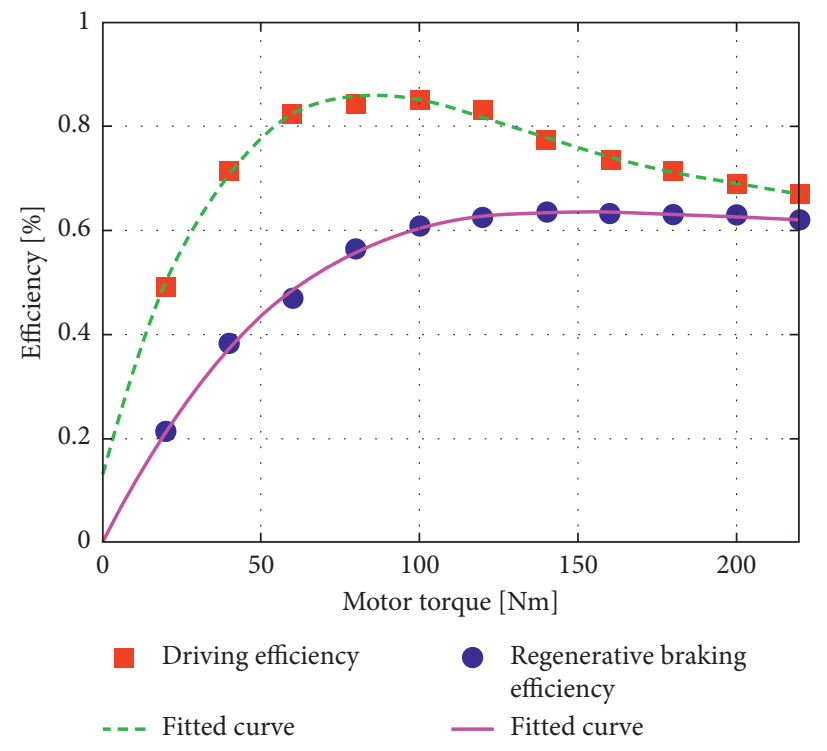

FIGURE 6: Driving efficiency and regenerative braking efficiency of EM. 


$$
\begin{aligned}
& \eta_{o}=\frac{T_{m} \omega}{P_{o}}, \\
& \eta_{i}=\frac{P_{i}}{T_{m} \omega},
\end{aligned}
$$

To analyze the proposed ECC scheme, the state-of-theart method of MPC-based cruise control is selected as a benchmark. The scheme of the benchmarked method is introduced in Figure 4(a) in Section 4.1. For the MPC in Figure 4(a), assuming $P$ is the predictive horizon, $M$ is the control horizon, and $M \leq P$, the predictive output sequence is $\widehat{Y}(k+1 / k)$ and the future control sequence $\Delta U(k)$ at sample time $k$ can be formulated as follows:

$$
\left\{\begin{array}{l}
\widehat{Y}\left(\frac{k+1}{k}\right)=\left[\begin{array}{llll}
\hat{y}(k+1) & \hat{y}(k+2) & \cdots & \hat{y}(k+P)
\end{array}\right]_{1 \times P}^{T}, \\
\Delta U(k)=\left[\begin{array}{llll}
\Delta u(k) & \Delta u(k+1) & \cdots & \Delta u(k+m-1)
\end{array}\right]_{1 \times M}^{T} .
\end{array}\right.
$$

In addition, the cost function $J$ can be constructed as

$$
J=\sum_{j=1}^{P}[\hat{y}(k+j)-y(k+j)]^{2}+\sum_{j=1}^{M} \lambda(j)[\Delta u(k+j-1)]^{2},
$$

where $\lambda$ is the weighting coefficient. The controller of MPC is used to make the cost function minimal. To obtain the optimum control sequence, we can make the derivative of the cost function equal to zero:

$$
\frac{\partial J}{\partial \Delta U}=0 .
$$

Then, the solution of $\Delta U(k)$ can be obtained. Moreover, by referring to the method in [23] and using the trial-anderror method, the prediction horizon length $M$ and the control horizon length $P$ are specified to be 10. The benchmarked cruise control and the proposed ECC are validated on the cosimulation platform of CarSim and Matlab/Simulink. With the cosimulation environment, the ECC and the benchmarked method are tested with different cruise speeds.

5.2. Results of Vehicle State Estimation. To analyze the performance of the improved BVCIE in Section 3, the test on the vehicle moving at $40 \mathrm{~km} / \mathrm{h}$ is presented. In addition, the recursive least square method with multiple forgetting factors (RLS-MFF), the extended Kalman filter (EKF), and the original method, i.e., BVCIE, are adopted for comparison.

For the original BVCIE, RLS-MFF, and EKF and the improved BVCIE, the initial values of the estimated vehicle total mass and pinch angle are set to 0 . The estimated total mass and the estimated pitch angle by the RLS-MFF, the $\mathrm{EKF}$, the original BVCIE, and the improved BVCIE are shown in Figures 7 and 8, respectively. In Figure 7, the blue curves represent the real value, the dotted black curves represent the estimated value by RLS-MFF, the dash-dotted green curves represent the estimated value by EKF, and the dashed orange curves represent the estimated value by the original BVCIE. From the results in Figure 7, especially the zoom-in curves in Figure 7, it can be found that the original BVCIE outperforms RLS-MFF and EKF in estimation accuracy. However, in case of the input data loss, the estimation results by RLS-MFF, EKF, and original BVCIE have a sharp wind-up. Figure 8 depicts the estimated results by the improved BVCIE. The blue curves represent the real value, and the dashed magenta curves represent the estimated value. From the results in Figure 8, we can find that the estimated values have good performances and can accurately reflect the real values. In addition, the wind-up phenomenon can be eliminated when the input data are lost. From the local zoom-in curves in Figures 8(a) and 8(b), we know that there exists a little error between the estimated value and the real value. To analyze the deviation, the estimation errors in total mass and in pitch angle by the improved BVCIE are shown in Figures 9(a) and 9(b). Compared with the estimated values in Figures 8(a) and 8(b), the estimation errors are very small. In this condition, the vehicle's state estimated by the method in Section 3 can be applied to the proposed ECC scheme in Section 4.

\section{Control Performances of ECC Scheme}

6.1. Control Performance at $40 \mathrm{~km} / \mathrm{h}$. To analyze the control performances of the proposed ECC and the benchmarked cruise control, the vehicle speed, the motor's torque (including the driving torque and the braking torque), and the motor's efficiency are analyzed in detail at $40 \mathrm{~km} / \mathrm{h}$ when moving on the test road. The results are shown in Figure 10. The blue curve and the red curve in Figure 10(a), respectively, reflect the vehicle speed by the benchmarked cruise control and ECC. The vehicle speed has a little perturbation when moving on the test road. On uphill part, the vehicle speed decreases less by ECC than by the benchmarked cruise control, and on downhill part, the vehicle speed increases less by ECC than by the benchmarked cruise control. Therefore, it can be obviously observed that ECC is better than the benchmarked cruise control in speed maintenance ability. From Figure 10(b), one can know that the motor's torques vary by different methods. Combining the speed profiles in Figure 10(a), we can find that the cruise speed by ECC has less fluctuation compared with that by the benchmarked cruise control, and most of the working points of the motor's torque by ECC are in the small value interval. Therefore, the motor's torque by ECC has less unreasonable actuation condition compared with that by the benchmarked cruise control. Figure 10(c) demonstrates the driving efficiency and the regenerative braking efficiency. According to the results in Figure 10 (c), we can observe that at some sampling instances the motor's efficiency by ECC is greater than that by the benchmarked cruise control, but at other sampling instances, the motor's efficiency by ECC is lower than that 


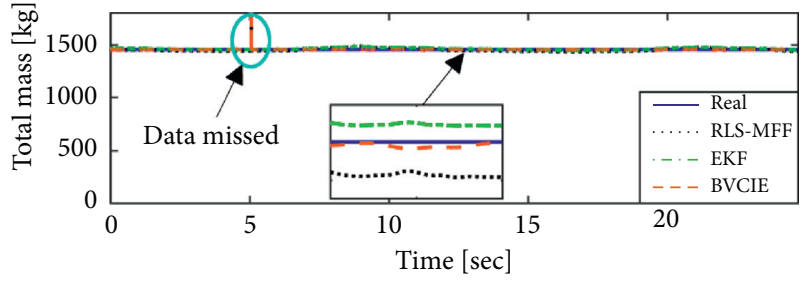

(a)

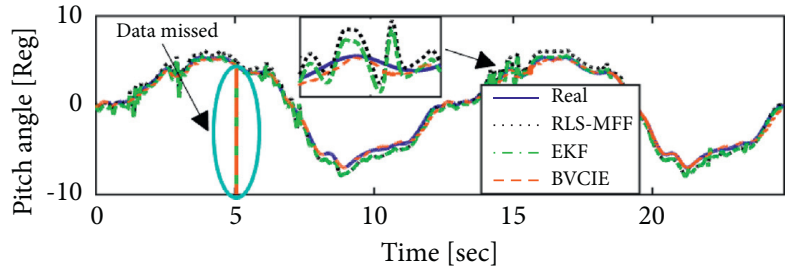

(b)

Figure 7: Estimation results by RLS-MFF, EKF, and BVCIE at $40 \mathrm{~km} / \mathrm{h}$ on asphalt road. (a) Estimated total mass. (b) Estimated pitch angle.

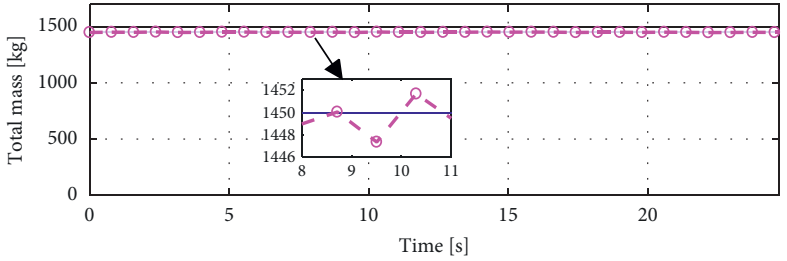

Real

$-\ominus-$ Improved BVCIE

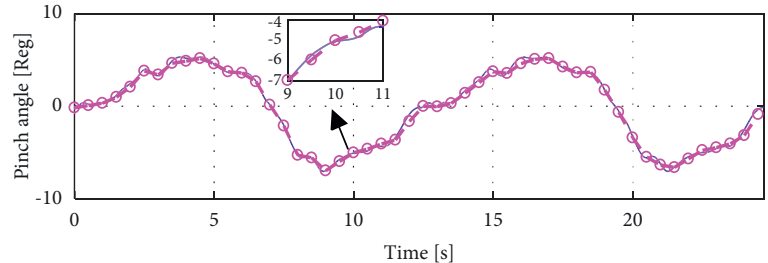

- Real

$-\ominus-$ Improved BVCIE

(a)

(b)

FIGURE 8: Estimation results by the improved BVCIE at $40 \mathrm{~km} / \mathrm{h}$ on asphalt road. (a) Estimated total mass. (b) Estimated pitch angle.

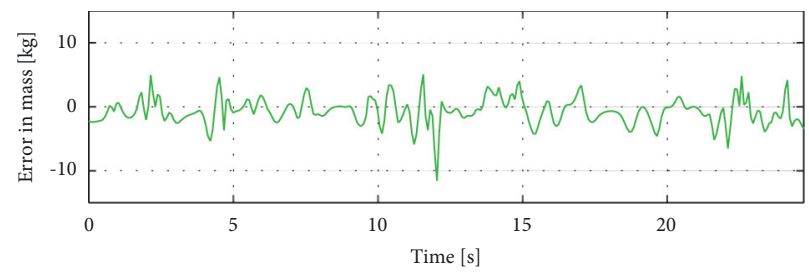

(a)

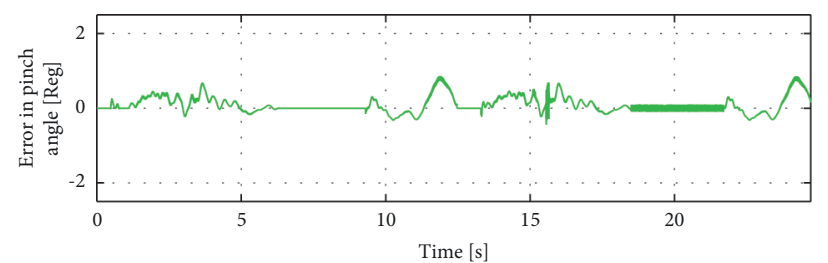

(b)

FIgURE 9: Estimation errors by the improved BVCIE at $40 \mathrm{~km} / \mathrm{h}$ on asphalt road. (a) Estimation error in mass. (b) Estimation error in pitch angle.

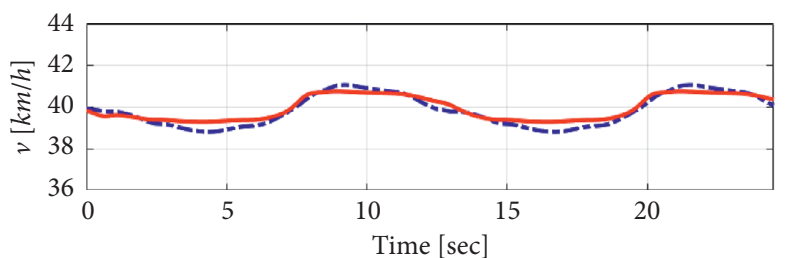

-..- Benchmark

— ECC

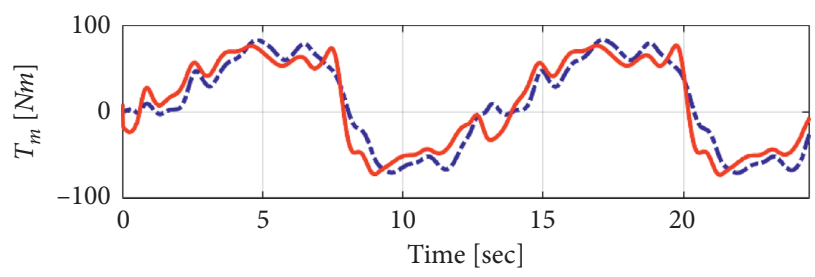

-..- Benchmark

- ECC

(a)

(b)

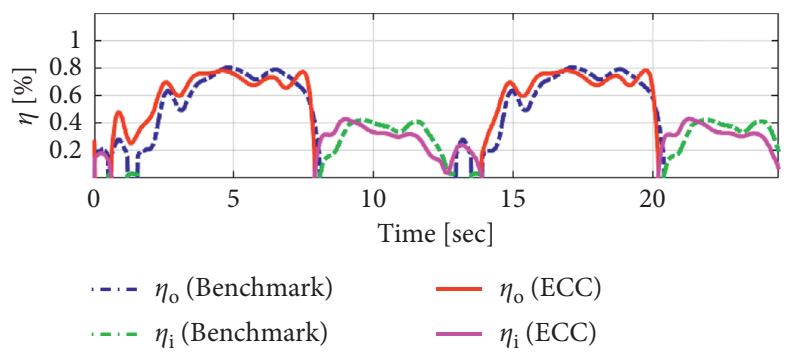

(c)

Figure 10: Control performances and comparison of ECC and benchmarked cruise control. (a) Vehicle speed. (b) Motor's torque. (c) Motor's efficiency. 
by the benchmarked cruise control. The reason for this phenomenon is that the motor drive system works in unreasonable actuation conditions at some sampling instances by the benchmarked cruise control. When the driving torque provided by the motor is smaller than the torque demands, the energy consumption by the benchmarked cruise control is less than that by ECC, but when the driving torque provided by the motor is greater than the torque demands, the energy consumption by the benchmarked cruise control is greater than that by ECC. In this condition, it is difficult to judge which method is better according to Figure $10(\mathrm{c})$. By referring to the analysis method in [41], the average driving efficiency and the average regenerative braking efficiency are introduced to compare the ECC and the benchmarked cruise control.

In addition, by referring to the analysis method in [42], the ride comfort can be assessed by vehicle's jerk $(J)$, which is the second derivative of the vehicle's longitudinal velocity. In the moving process, the jerk values include positive values and negative values. For this reason, we adopt the average absolute jerk to compare the ride comfort of ECC and the benchmarked cruise control. The smaller the average absolute jerk, the better the ride comfort. The average absolute jerk is defined as

$$
|\bar{J}|=\frac{1}{N} \sum_{k=1}^{N}|\ddot{v}(k)| .
$$

The average driving efficiency, the average regenerative braking efficiency, and the average absolute jerk are shown in Table 2.

According to the results in Table 2, in the driving mode, the average driving efficiency by the benchmarked cruise control is significantly lower than that by ECC. In the braking mode, the average driving efficiency by ECC is similar to that by the benchmarked cruise control. Thus, ECC reasonably adjusts the motor's toque so that the motor works in the high efficiency range. In addition, the average absolute jerk by ECC is smaller than that by the benchmarked cruise control. From the results in Table 2, we can conclude that the motor's efficiency and the ride comfort by ECC outperform those by the benchmarked cruise control in the driving mode and braking mode.

6.2. Control Performance at $80 \mathrm{~km} / \mathrm{h}$. To demonstrate the performance of the proposed ECC at high cruise speed, the cruise speed of $80 \mathrm{~km} / \mathrm{h}$ is investigated in this part. The vehicle speed, the motor's torque, and the motor's efficiency are presented in Figure 11. In Figure 11(a), it is obvious that the speed maintenance ability by ECC is still more excellent than that by the benchmarked cruise control. Figures 11(b) and $11(\mathrm{c})$, respectively, demonstrate the motor's torque and the motor's efficiency. Similar to the results in Figures 11(b) and $11(\mathrm{c})$, it is still difficult to distinguish which method is better. That is because sometimes the motor's torque and the motor's efficiency by ECC are greater than those by the benchmarked cruise control, but sometimes the motor's torque and the motor's efficiency by ECC are lower than
TABle 2: Motor's average efficiency and ride comfort by the benchmarked cruise control and the proposed ECC $(40 \mathrm{~km} / \mathrm{h})$.

\begin{tabular}{|c|c|c|}
\hline Control performances & Benchmark & ECC \\
\hline Driving efficiency & 0.5827 & $0.6468 \times 100 \%$ \\
\hline Regenerative braking efficiency & 0.2790 & $0.2900 \times 100 \%$ \\
\hline Average absolute jerk & 1.3964 & $1.1930 \mathrm{~km} / \mathrm{s}^{2}$ \\
\hline
\end{tabular}

those by the benchmarked cruise control. Therefore, we also calculate the average driving efficiency, the average regenerative braking efficiency, and the average absolute jerk, and they are shown in Table 3.

From Table 3, it can be found that in the driving mode, the driving efficiency by ECC is higher than that by the benchmarked cruise control. More importantly, the regenerative braking efficiency by ECC is also higher than that by the benchmarked cruise control. In addition, the average absolute jerk by ECC is still smaller than that by the benchmarked cruise control. Therefore, it can be concluded that ECC is better than the benchmarked cruise control at high cruise speed.

\section{Energy Saving Performance Analysis}

To demonstrate vehicle's energy saving performance of the proposed ECC, the energy consumption of the two scenarios in Sections 6.1 and 6.2 is analyzed in detail. The energy consumption in the driving mode and the energy feedback in the braking mode are calculated as follows:

$$
\begin{aligned}
& E_{o}=\int_{0}^{t_{f}} P_{o}(t) \mathrm{d} t, \\
& E_{i}=\int_{0}^{t_{f}} P(t) \mathrm{d} t_{i},
\end{aligned}
$$

where $E_{o}$ refers to the energy consumption in the driving mode, $E_{i}$ refers to the energy feedback in the braking mode, and $t_{f}$ refers to the termination time.

The energy consumption and energy feedback of the two scenarios in Sections 6.1 and 6.2 are shown in Figures 12(a) and $12(\mathrm{~b})$, respectively. When the test vehicle is moving at $40 \mathrm{~km} / \mathrm{h}$, the energy consumption by the ECC and that by the benchmarked cruise control are $0.2033 \mathrm{kwh}$ and $0.1956 \mathrm{kwh}$, respectively. The energy feedback by ECC and the benchmarked cruise control is $0.0424 \mathrm{kwh}$ and $0.0496 \mathrm{kwh}$, respectively. Obviously, it can be found that the vehicle consumes less energy by ECC when moving at $40 \mathrm{~km} / \mathrm{h}$ compared with that by the benchmarked cruise control. For the high cruise speed of $80 \mathrm{~km} / \mathrm{h}$, the energy consumption by the ECC and by the benchmarked cruise control is $0.1709 \mathrm{kwh}$ and $0.1583 \mathrm{kwh}$, respectively. In addition, the energy feedback by the ECC and by the benchmarked cruise control is $0.0472 \mathrm{kwh}$ and $0.0483 \mathrm{kwh}$. The results in Figure 12(b) show the advantage of ECC because the vehicle not only consumes less energy in the driving mode, but also regenerates more energy in the braking mode.

For the two scenarios, the real energy consumption $(E)$ in the whole process can be calculated as 


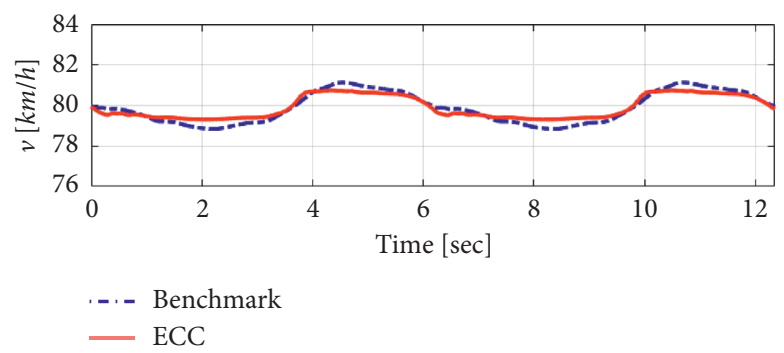

(a)

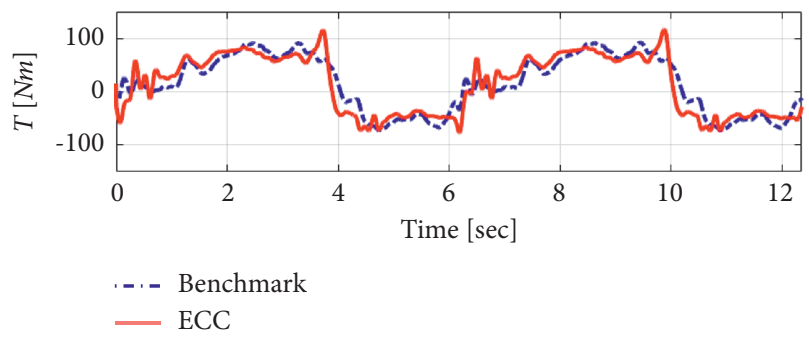

(b)

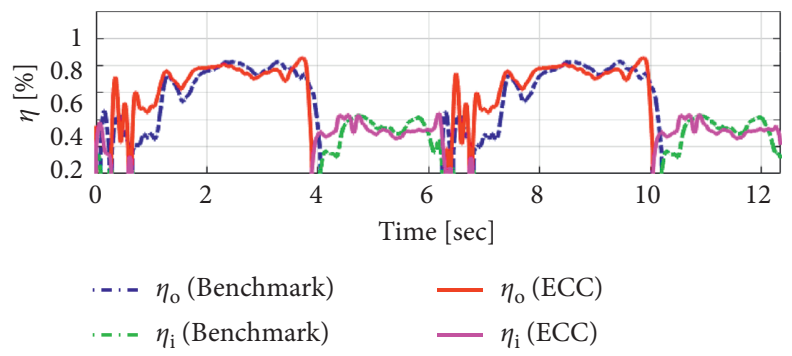

(c)

Figure 11: Control performances and comparison of ECC and benchmarked cruise control. (a) Vehicle speed. (b) Motor's torque. (c) Motor's efficiency.

TABLE 3: Motor's average efficiency and ride comfort by the benchmarked cruise control and the proposed ECC ( $80 \mathrm{~km} / \mathrm{h})$.

\begin{tabular}{|c|c|c|}
\hline Control performances & Benchmark & ECC \\
\hline Driving efficiency & 0.6103 & $0.6775 \times 100 \%$ \\
\hline Regenerative braking efficiency & 0.2973 & $0.3057 \times 100 \%$ \\
\hline Average absolute jerk & 1.4265 & $1.2646 \mathrm{~km} / \mathrm{s}^{2}$ \\
\hline
\end{tabular}

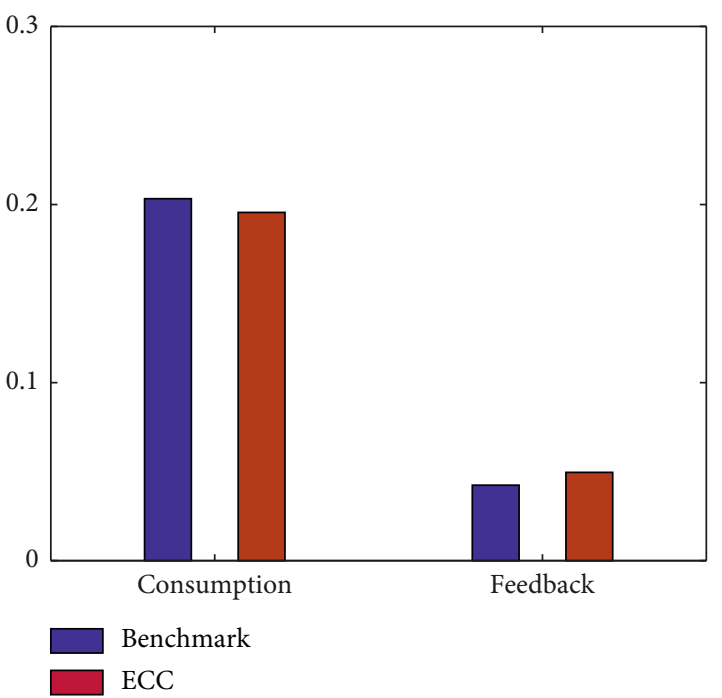

(a)

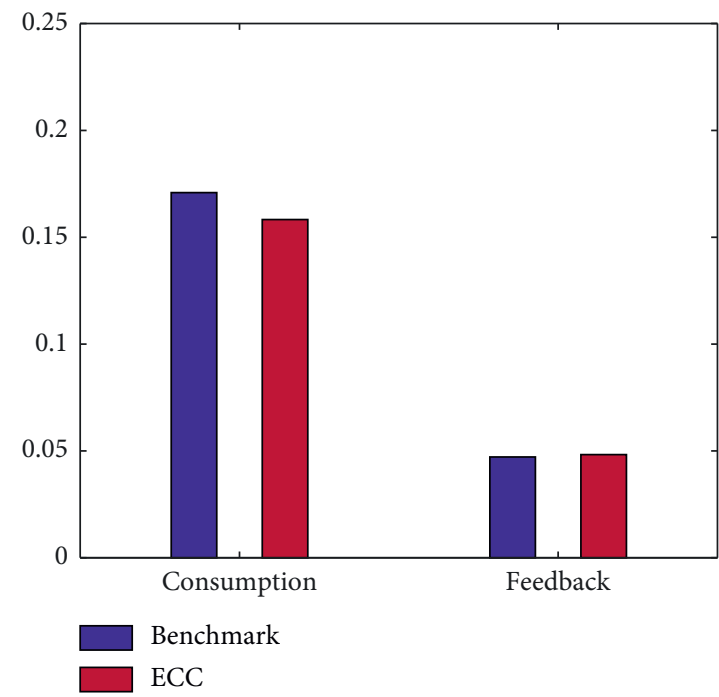

(b)

FIgURE 12: Results of energy consumption and feedback (kwh). (a) At $40 \mathrm{~km} / \mathrm{h}$. (b) At $80 \mathrm{~km} / \mathrm{h}$.

$$
E=E_{o}-E_{i}
$$

To analyze the real energy consumption on the test road in detail, the cruise speeds of $40 \mathrm{~km} / \mathrm{h}, 50 \mathrm{~km} / \mathrm{h}, 60 \mathrm{~km} / \mathrm{h}, 70 \mathrm{~km} / \mathrm{h}$, $80 \mathrm{~km} / \mathrm{h}, 90 \mathrm{~km} / \mathrm{h}, 100 \mathrm{~km} / \mathrm{h}$, and $110 \mathrm{~km} / \mathrm{h}$ are selected to investigate ECC performance, and the proposed ECC is detailedly compared with the benchmarked cruise control. The total energy consumption on the test road at $40 \mathrm{~km} / \mathrm{h}, 50 \mathrm{~km} / \mathrm{h}$, $60 \mathrm{~km} / \mathrm{h}, 70 \mathrm{~km} / \mathrm{h}, 80 \mathrm{~km} / \mathrm{h}, 90 \mathrm{~km} / \mathrm{h}, 100 \mathrm{~km} / \mathrm{h}$, and $110 \mathrm{~km} / \mathrm{h}$ is shown in Figure 13. It is obvious that the test vehicle 


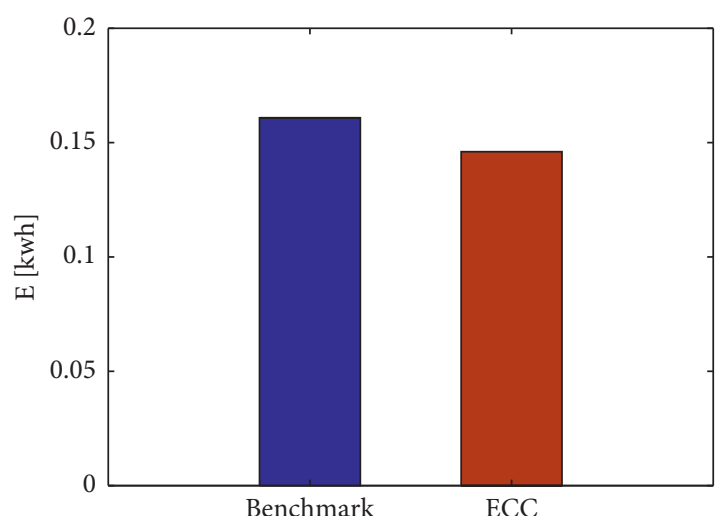

(a)

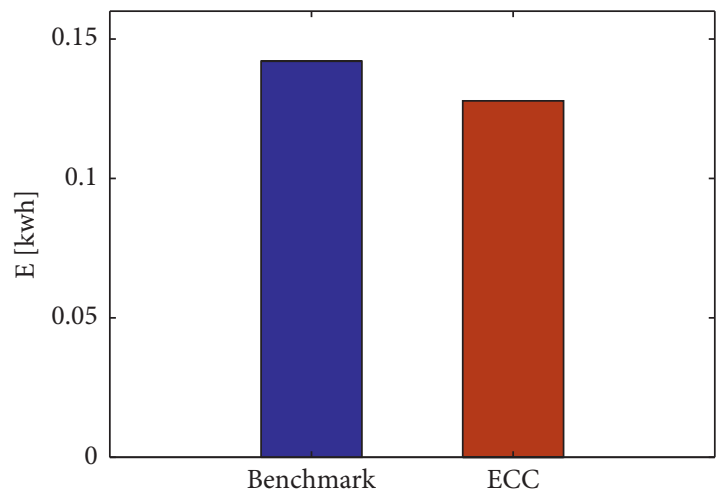

(c)

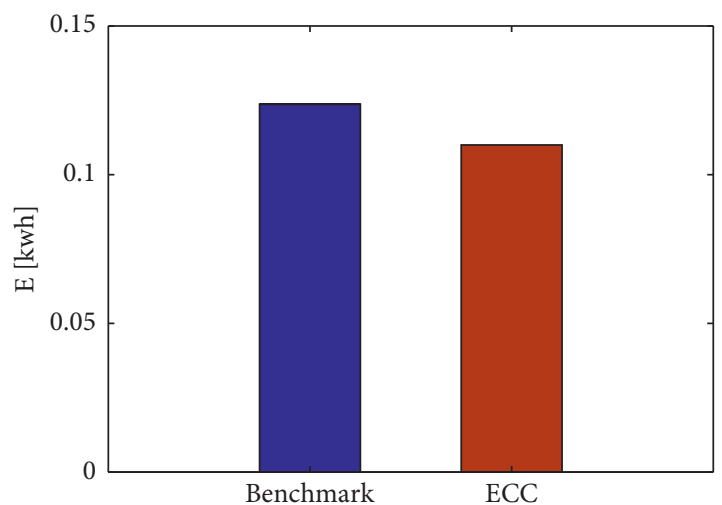

(e)

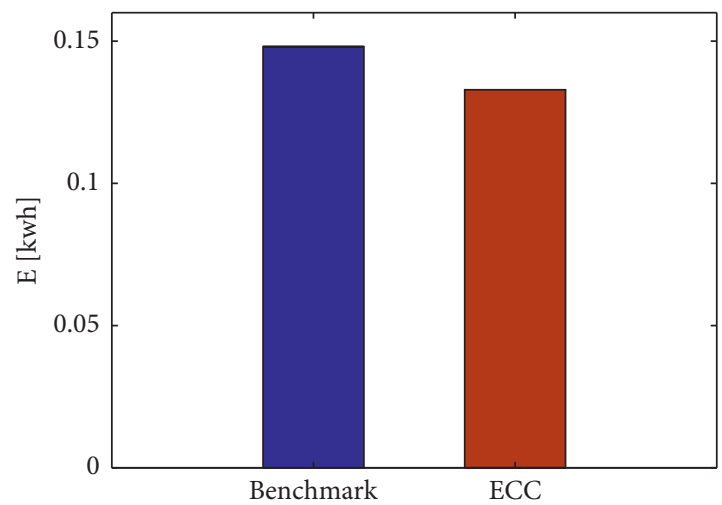

(g)

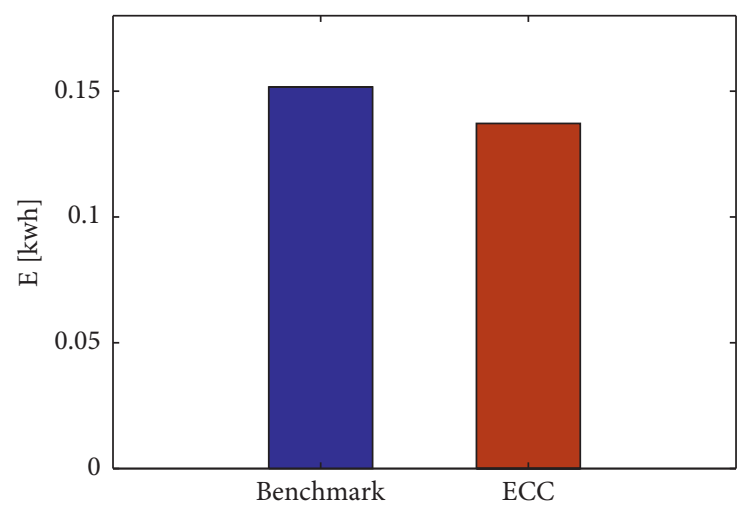

(b)

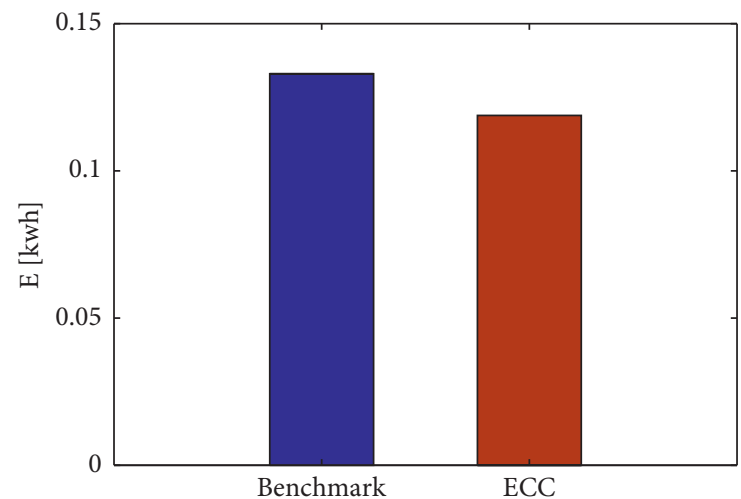

(d)

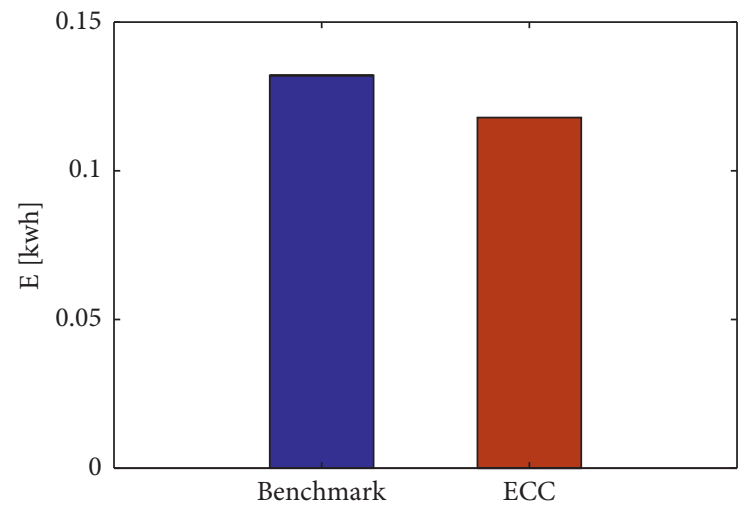

(f)

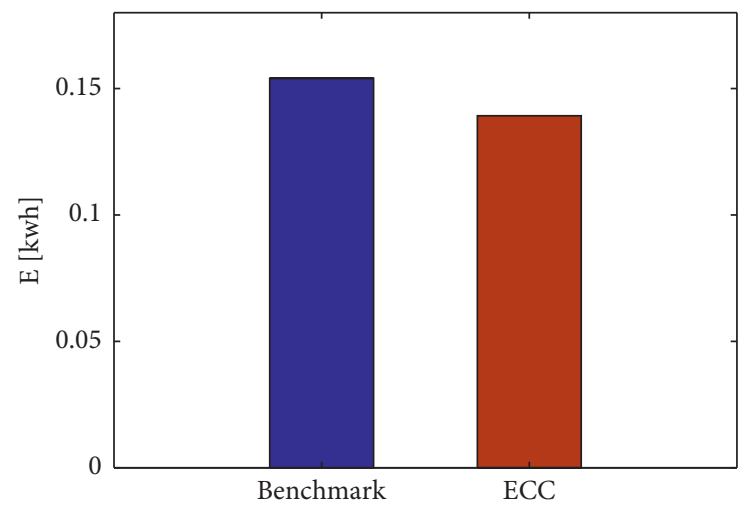

(h)

Figure 13: Energy consumption at different cruise speeds. (a) At $40 \mathrm{~km} / \mathrm{h}$. (b) At $50 \mathrm{~km} / \mathrm{h}$. (c) At $60 \mathrm{~km} / \mathrm{h}$. (d) At $70 \mathrm{~km} / \mathrm{h}$. (e) At $80 \mathrm{~km} / \mathrm{h}$. (f) At $90 \mathrm{~km} / \mathrm{h}$. (g) At $100 \mathrm{~km} / \mathrm{h}$. (h) At $110 \mathrm{~km} / \mathrm{h}$. 


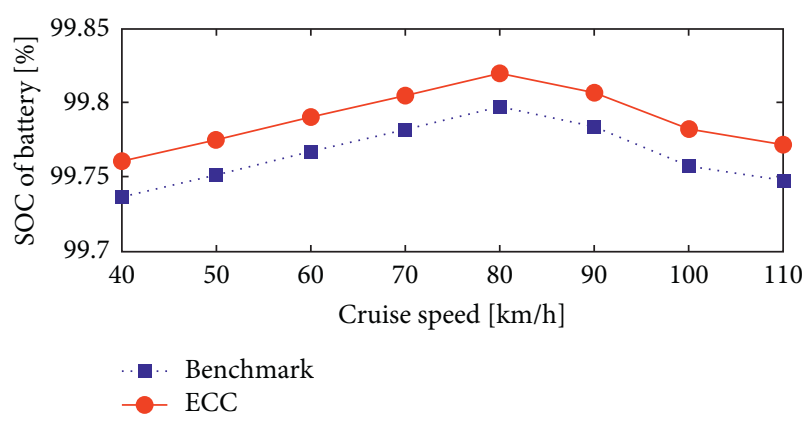

FIgURE 14: Battery SOC at different cruise speeds.

consumes less energy in all cruise speeds by ECC compared with that by the benchmarked cruise control. The results in Figure 13 effectively prove that the proposed ECC has better performances in decreasing the energy consumption.

Furthermore, to compare the energy saving performance of the proposed ECC and the benchmarked cruise control, the state of charge (SOC) of the battery is adopted. Before implementing the simulation, the battery SOC is $100 \%$. After the simulation, the battery SOC at $40 \mathrm{~km} / \mathrm{h}, 50 \mathrm{~km} / \mathrm{h}, 60 \mathrm{~km} / \mathrm{h}$, $70 \mathrm{~km} / \mathrm{h}, 80 \mathrm{~km} / \mathrm{h}, 90 \mathrm{~km} / \mathrm{h}, 100 \mathrm{~km} / \mathrm{h}$, and $110 \mathrm{~km} / \mathrm{h}$ is shown in Figure 14. The dotted blue line refers to the battery SOC by the benchmarked cruise control, and the solid red line refers to the battery SOC by the proposed ECC. It can be easily found that the battery SOC by the proposed method is higher than that by the benchmarked cruise control. Therefore, the proposed ECC outperforms the benchmarked cruise control in energy saving performance.

\section{Conclusion}

Motivated by the driving range limits of electric vehicles, this paper proposes an ECC to improve the energy utilization efficiency of electric vehicles. Different from the prevailing research, this paper focuses on the condition that the electric vehicle is moving on the slope road without using the digital map's information and the GPS information. Additionally, energy saving is realized by reducing the unreasonable actuation of the motor's torque. Compared with the benchmarked cruise control, the proposed ECC not only improves the electric vehicle's speed maintenance ability and ride comfort, but also improves the energy efficiency. Moreover, different cruise speed tests indicate that the proposed ECC can obviously decrease the energy consumption, and the statistical results of the battery SOC show that the proposed ECC can improve the energy saving performance. The research in this paper provides a novel method to improve electric vehicles' energy efficiency.

\section{Data Availability}

The data used to support the findings of this study are available from the corresponding author upon request.

\section{Conflicts of Interest}

The authors declare that there are no conflicts of interest regarding the publication of this paper.

\section{Acknowledgments}

This work was supported in part by the National Key R\&D Program of China (2018YFB2101304), the Fundamental Research Funds for the Central Universities (3102020QD1004), the Natural Science Foundation of Shaanxi Province (2021JQ121), the National Natural Science Foundation of China (51674113 and 62106202), and the Joint Aeronautical Science Foundation Project (202051053002).

\section{References}

[1] Z. Q. Liu, T. Zhang, and Y. F. Wang, "Research on local dynamic path planning method for intelligent vehicle lanechanging," Journal of Advanced Transportation, vol. 43, no. 1, pp. 47-70, 2019.

[2] F. Farivar, M. S. Haghighi, A. Jolfaei et al., "Covert attacks through adversarial learning: studying the effect of lane keeping attacks on the safety of autonomous vehicles," IEEE, vol. 26, no. 3, pp. 1350-1357, 2021.

[3] S. Rafatnia and M. Mirzaei, "Adaptive estimation of vehicle velocity from updated dynamic model for control of anti-lock braking system," IEEE Transactions on Intelligent Transportation Systems, 2021.

[4] Z. Liu, Q. Yuan, G. Nie et al., "A multi-objective model predictive control for vehicle adaptive cruise control system based on a new safe distance model," International Journal of Automotive Technology, vol. 22, no. 2, pp. 475-487, 2021.

[5] L. Zhang, Y. Wang, and Z. Wang, "Robust lateral motion control for in-wheel-motor-drive electric vehicles with network induced delays," IEEE Transactions on Vehicular Technology, vol. 68, no. 11, pp. 10585-10593, 2019.

[6] L. Zhang, Z. Wang, X. Ding et al., "Fault-tolerant control for intelligent electrified vehicles against front wheel steering angle sensor faults during trajectory tracking," IEEE Access, vol. 9, pp. 65174-65186, 2021.

[7] X. Ding, Z. Wang, and L. Zhang, "Hybrid control-based acceleration slip regulation for four-wheel-independentlyactuated electric vehicles," IEEE Transactions on Transportation Electrification, vol. 7, no. 3, pp. 1976-1989, 2020.

[8] Y. Zhang, Y. Zhang, Z. Ai et al., "Energy optimal control of motor drive system for extending ranges of electric vehicles," IEEE Transactions on Industrial Electronics, vol. 68, no. 2, pp. 1728-1738, 2021.

[9] G. Q. Song, Y. Yang, H. Hang et al., "Study on vehicle collision-avoiding radar and intelligent cruise control system," Key Engineering Materials, vol. 297-300, pp. 311-315, 2005.

[10] A. Swetha, N. R. A. Asmy, V. R. Pillay et al., "Algorithm for autonomous cruise control system - real time scheduling and control aspects," Advances in Intelligent Systems \& Computing, vol. 327, pp. 449-456, 2014.

[11] X. W. Chen, J. G. Zhang, and Y. J. Liu, "Research on the intelligent control and simulation of automobile cruise system based on fuzzy system," Mathematical Problems in Engineering, vol. 2016, Article ID 9760653, 12 pages, 2016.

[12] K. Li, UkawaHiroshi, and HandaMasatoshi, "Nonlinear disturbance decoupling control of heavy-duty truck stop and go cruise system," Vehicle System Dynamics, vol. 47, no. 1, pp. 29-55, 2009.

[13] N. Vedam, I. Diaz-Rodrigue, and S. P. Bhattacharyya, "A novel approach to the design of controllers in an automotive cruise-control system," in Proceedings of the IECON 2014-40th Annual Conference of the IEEE Industrial 
Electronics Society, pp. 2927-2932, Dallas, TX, USA, February 2015.

[14] M. Laheeb, K. Salwan, A. B. Fawaz et al., "Online algorithm for controlling a cruise system under uncertainty in design parameters and environmental conditions using Monte-Carlo simulation," in Proceedings of the 2018 IEEE 8th Annual Computing and Communication Workshop and Conference (CCWC), pp. 424-430, Las Vegas, NV, USA, February 2018.

[15] L. Cai, A. B. Rad, and W. L. Chan, "An intelligent longitudinal controller for application in semiautonomous vehicles," IEEE Transactions on Industrial Electronics, vol. 57, no. 4, pp. 1487-1497, 2010.

[16] A. Swetha, S. Dasgupta, S. K. Chandran et al., "A real-time performance monitoring tool for dual redundant and resource augmented framework of cruise control system," International Journal of Computer Applications in Technology, vol. 92, no. 14, pp. 44-49, 2014.

[17] A. Swetha, P. V. Radhamani, and S. Punnekkat, "Design, analysis and implementation of improved adaptive fault tolerant model for cruise control multiprocessor system," International Journal of Computer Applications in Technology, vol. 86 , no. 15 , pp. 29-26, 2014.

[18] P. Nilsson, O. Hussien, A. Balkan et al., "Correct-by-construction adaptive cruise control:two approaches," IEEE Transactions on Control Systems Technology, vol. 24, no. 4, pp. 1294-1307, 2016.

[19] S. H. Hosseinnia, I. Tejado, V. Milanés et al., "Experimental application of hybrid fractional-order adaptive cruise control at low speed," IEEE Transactions on Control Systems Technology, vol. 22, no. 6, pp. 2329-2336, 2014.

[20] K. C. Dey, L. Yan, X. Wang et al., "A review of communication, driver characteristics, and controls aspects of cooperative adaptive cruise control (CACC)," IEEE Transactions on Intelligent Transportation Systems, vol. 17, no. 2, pp. 491-509, 2016.

[21] F. Li and Y. Wang, "Cooperative adaptive cruise control for string stable mixed traffic: benchmark and human-centered design," IEEE Transactions on Intelligent Transportation Systems, vol. 18, no. 12, pp. 3473-3485, 2017.

[22] S. Öncü, J. Ploeg, N. Van de Wouw et al., "Cooperative adaptive cruise control: network-aware analysis of string stability," IEEE Transactions on Intelligent Transportation Systems, vol. 15, no. 4, pp. 1527-1537, 2014.

[23] C. M. Filho, M. H. Terra, and D. F. Wolf, "Safe optimization of highway traffic with robust model predictive control-based cooperative adaptive cruise control," IEEE Transactions on Intelligent Transportation Systems, vol. 18, no. 11, pp. 3193-3203, 2017.

[24] B. V. Arem, C. J. G. V. Driel, and R. Visser, "The impact of cooperative adaptive cruise control on traffic-flow characteristics," IEEE Transactions on Intelligent Transportation Systems, vol. 7, no. 4, pp. 429-436, 2006.

[25] B. Liu and A. E. Kamel, "V2X-based decentralized cooperative adaptive cruise control in the vicinity of intersections," IEEE Transactions on Intelligent Transportation Systems, vol. 17, no. 3, pp. 644-658, 2016.

[26] Y. Zhang, Y. Zhang, Z. Ai et al., "Energy saving control strategy for the high-frequency start-up process for electric mining haul trucks," IEEE Transactions on Intelligent Vehicles, vol. 3, no. 4, pp. 595-606, 2018.

[27] B. Alrifaee, J. G. Jodar, and D. Abel, "Decentralized predictive cruise control for energy saving in REEV using V2I information for multiple-vehicles," IFAC Papersonline, vol. 48, no. 15, pp. 320-327, 2015.
[28] B. Alrifaee, Y. Liu, and D. Abel, "Eco-cruise control using economic model predictive control," in Proceedings of the IEEE Conference on Industrial Automation and Control Emerging Technology Applications, pp. 1933-1938, Sydney, Australia, September 2015.

[29] B. Alrifaee, J. G. Jodar, and D. Abel, "Predictive cruise control for energy saving in REEV using V2I information," in Proceedings of the 2015 23rd Mediterranean Conference on Control and Automation (MED), pp. 82-87, Torremolinos, Spain, July 2015.

[30] B. Asadi and A. Vahidi, "Predictive cruise control: utilizing upcoming traffic signal information for improving fuel economy and reducing trip time," IEEE Transactions on Control Systems Technology, vol. 19, no. 3, pp. 707-714, 2011.

[31] H. Yang, H. Rakha, and M. V. Ala, "Eco-cooperative adaptive cruise control at signalized intersections considering queue effects," IEEE Transactions on Intelligent Transportation Systems, vol. 18, no. 6, pp. 1575-1585, 2017.

[32] E. Talavera, A. Díaz-Álvarez, F. Jiménez et al., "Impact on congestion and fuel consumption of a cooperative adaptive cruise control system with lane-level position estimation," Energies, vol. 11, no. 1, p. 194, 2018.

[33] Y. Chen, X. Li, C. Wiet et al., "Energy management and driving strategy for in-wheel motor electric ground vehicles with terrain profile preview," IEEE Transactions on Industrial Informatics, vol. 10, no. 3, pp. 1938-1947, 2014.

[34] M. A. S. Kamal et al., "Ecological vehicle control on roads with up-down slopes," IEEE Transactions on Intelligent Transportation Systems, vol. 12, no. 3, pp. 783-794, 2011.

[35] R. T. Bambang, A. S. Rohman, C. J. Dronkers et al., "Energy management of fuel cell/battery/supercapacitor hybrid power sources using model predictive control," IEEE Transactions on Industrial Informatics, vol. 10, no. 4, pp. 1992-2002, 2014.

[36] D. Tian, Y. Zhu, X. Duan et al., "An effective fuel level data cleaning and repairing method for vehicle monitor platform," IEEE Transactions on Industrial Informatics, vol. 15, no. 1, pp. 410-422, 2019.

[37] Y. Zhang, Y. Zhang, Z. Ai et al., "A cross iteration estimator with base vector for estimation of electric mining haul truck's mass and road grade," IEEE Transactions on Industrial Informatics, vol. 44, no. 9, pp. 4138-4148, 2018.

[38] Y. Zhang, Y. Zhang, Z. Ai et al., "Study on electric sunroof pinch detection of cars based on model reference adaptive cholesky decomposition filter," IEEE Transactions on Transportation Electrification, vol. 4, no. 1, pp. 190-201, 2018.

[39] A. Weißmann, D. Görges, and X. Lin, "Energy-optimal adaptive cruise control combining model predictive control and dynamic programming," Control Engineering Practice, vol. 72, pp. 125-137, 2018.

[40] Sajadi-Alamdari, S. Amin, H. Voos, and M. Darouach, "Nonlinear model predictive extended eco-cruise control for battery electric vehicles," in Proceedings of the 24th Conference on Control and Automation, pp. 467-472, Athens, Greece, June 2016.

[41] Y. Chen and J. Wang, "Design and experimental evaluations on energy efficient control allocation methods for overactuated electric vehicles: longitudinal motion case," IEEE, vol. 19, no. 2, pp. 538-548, 2014.

[42] C. Lv, X. Hu, A. Sangiovanni-Vincentelli et al., "Driving-stylebased co-design optimization of an automated electric vehicle: a cyber-physical system approach," IEEE Transactions on Industrial Electronics, vol. 66, no. 4, pp. 2965-2975, 2018. 